\title{
Image Galleries for DNA Ploidy Measurement: a Tool for Screening, Learning, Data and Image Processing
}

\author{
Paulette Herlin $\left({ }^{1}\right)$, David Deman $\left({ }^{2}\right)$, Christophe Boudry $\left({ }^{1,3,4}\right)$, François Angot $\left({ }^{5}\right)$ \\ and Françoise Duigou $\left({ }^{1}\right)$ \\ $\left({ }^{1}\right)$ Service d'anatomie pathologique, Centre F. Bàclesse, 14076 Caen, France \\ $\left({ }^{2}\right)$ ENSI, ISMRA 14050 Caen Cedex, France ${ }^{\circ}$ \\ $\left({ }^{3}\right)$ LERMAT, URA CNRS 1317, ISMRA, 14050 Caen Cedex, France \\ $\left({ }^{4}\right)$ CYCERON, Université de Caen, URA CNRS 1829, 14074 Caen Cedex, France \\ $\left({ }^{5}\right)$ GREYC, URA CNRS 1526, ISMRA, 14050 Caen Cedex, France
}

\begin{abstract}
Résumé. - Déjà proposée par quelques analyseurs du commerce, l'affichage des galeries d'imagettes offre au pathologiste le confort d'un contrôle visuel, a posteriori, de la qualité d'un étiquetage automatique des éléments cellulaires analysés. Nous avons tenté d'exploiter au maximum cet outil pour la mesure automatique de l'ADN ploïdie des tumeurs et d'étendre son utilisation à la mise au point de stratégies d'analyse d'images. Le détail de ces fonctionnalités fait l'objet du présent article : les imagettes offrent de nouveaux outils d'apprentissage et d'aide au tri, de screening et de contrôle de la qualité du tri, de retraitement des données et de traitement d'images.
\end{abstract}

\begin{abstract}
Display of image galleries offers to pathologists the confort of an a posteriori visual inspection of the quality of automatic cell classification. Some commercially available systems have already developed this functionality. In an attempt to facilitate the post-processing of automatically acquired DNA ploidy data, this tool was exploited and its use extended to the elaboration and test of new image analysis strategies. Details of this new functionnalities are presented: image galleries offers tools for learning, screening and quality control, data post-processing and image processing.
\end{abstract}

\section{Introduction}

DNA ploidy is able to provide clinicians with additional information concerning the potential evolution of tumors $[1,2]$. The wide spread use in clinical oncology of image analysers devoted to this task, needs to develop fully automated procedures (image acquisition, object segmentation, cell sorting and data post-processing). This complete automation is the only way to collect a meaningful cell population and to give statistically significant results in an acceptable delay. Nevertheless, the pathologist will appreciate to benefit of an a posteriori visual control of the result of automatic labelling. This comfort requires the creation, manipulation and visualization of small 
images corresponding to independent entities generated from original images, and strictly linked to the corresponding measures. The introduction of this new functionality in the laboratory was the starting point of the discover of several other potential applications of this tool, which will be here detailed.

\section{Material and Methods}

DNA ploidy measurement is performed on microscopic preparations of cell nuclei, isolated from paraffin embedded tumors [3]. Image acquisition $512 \times 512$ pixels, 256 grey levels, is done at a resolution of $0.11 \mu \mathrm{m}^{2}$ thanks to a B.W. C.C.D. camera (Sony), a motorized BH2 microscope (Olympus) and a P.I.P. 1024 frame grabber (Matrox). The automatic measurement of DNA ploidy abnormalities is done thanks to DRACCAR ${ }^{\circledR}$ software (Gestinfor Corp., Caen, and ADCIS Corp., Caen, France). Small images are generated one by one from original images, after the calculation of Feret diameters and coordinates of each segmented objects (3000 to 5000 small images per case). Their original serial number provides the bridge with the corresponding 40 measured parameters (size, shape, intensity, texture, integrated optical density and corresponding DNA value).

\section{Taking Advantages of Small Images}

Tool for Training And Help for Interactive Sorting. - The automatic labelling of nuclei to be measured is done by reference to a specific knowledge base built interactively. The progressive enrichment of this base is promoted by the visualization of typical examples of cell nuclei and the visualization of nuclei corresponding to allowed morphological margins for each class (Fig. 1).

Tool for Screening, Sorting Quality Control and Refinement of Knowledge BASE BUILDING. - The a posteriori display of the comprehensive view of the whole small images as a gallery corresponding to each class, together with the ability of modifying cell group, object per object, offers several advantages (Fig. 1). It allows correction of automatic or interactive mislabelling. The mistakes are more easy to detect as the gallery offers the visualization of morphological homogeneous groups, as opposed to the original image. It is a way to save time and improve sorting quality. Furthermore, small images provide experts with a simple tool for testing reproducibility of sorting from the same set of images. An inter or intra expert control gives rise to data collection, free of all uncertainty of sorting. This new tool was very recently introduced in the laboratory for routine use and we are up to now unable to give statistically valuable results regarding cell classification reproducibility.

Tool for Data Post-Processing. - The strict correspondence established between measured parameters and small images allows to use them as data post-processing adjuncts. For example, one is able to display galleries of nuclei corresponding to any selected region of interest of a DNA ploidy histogram (Fig. 2) or of any couple of parameter 2D map. This allows to establish an interrelationship between nuclear morphology disturbances and DNA ploidy abnormalities. The interactive projection of the coordinates of any small image on any couple of parameter 2D map permits to statistically delineate families of nuclei, using the dynamic clustering method (Fig. 3). Dots on 2D map, corresponding to coordinates of typical nuclei identified on galleries by the expert, can be used as starting germs. 


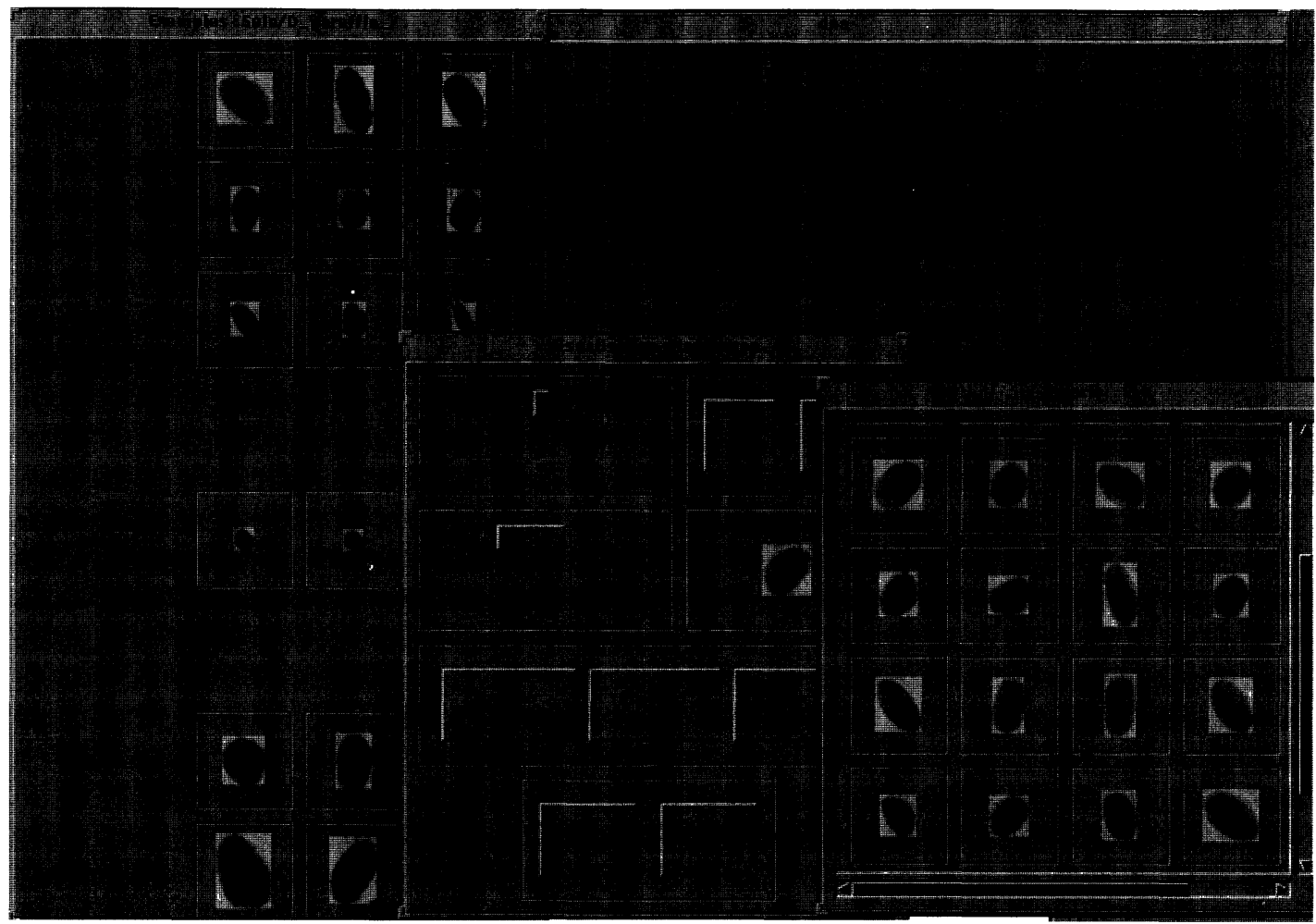

Fig. 1. - Visualization of labelled nuclei thanks to image galleries with modification of label facilities.
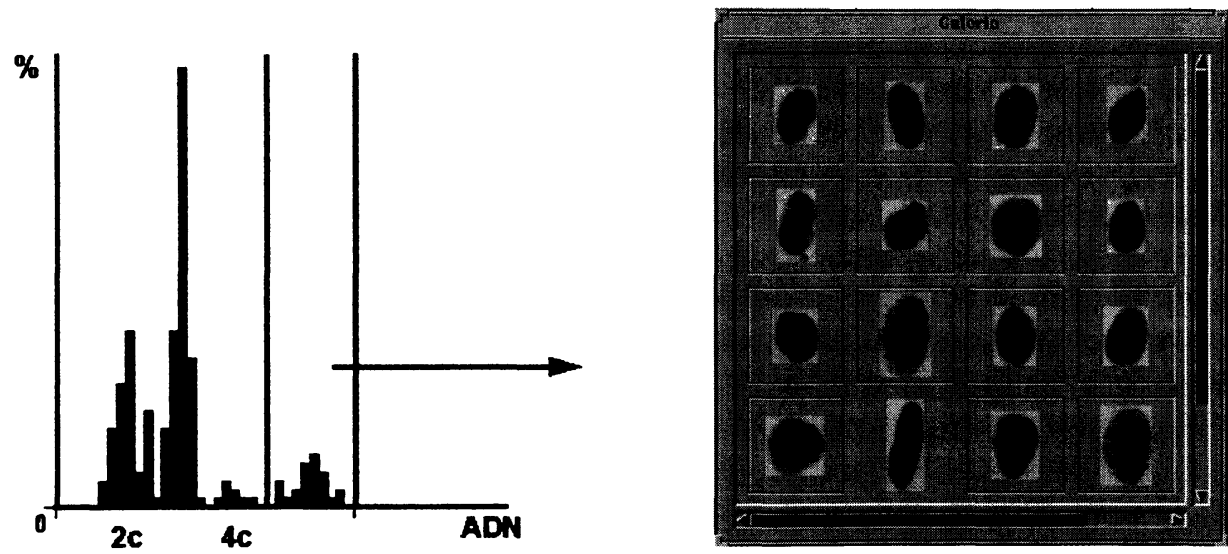

Fig. 2. - Gating on DNA ploidy histogram and display of the corresponding nuclei.

Tool for Image Treatment. - Small images, which are 5 to 10 times less bulky than the corresponding initial images, and which are organized in cell groups can be used for rapid testing of new image analysis treatment (Fig. 4). 


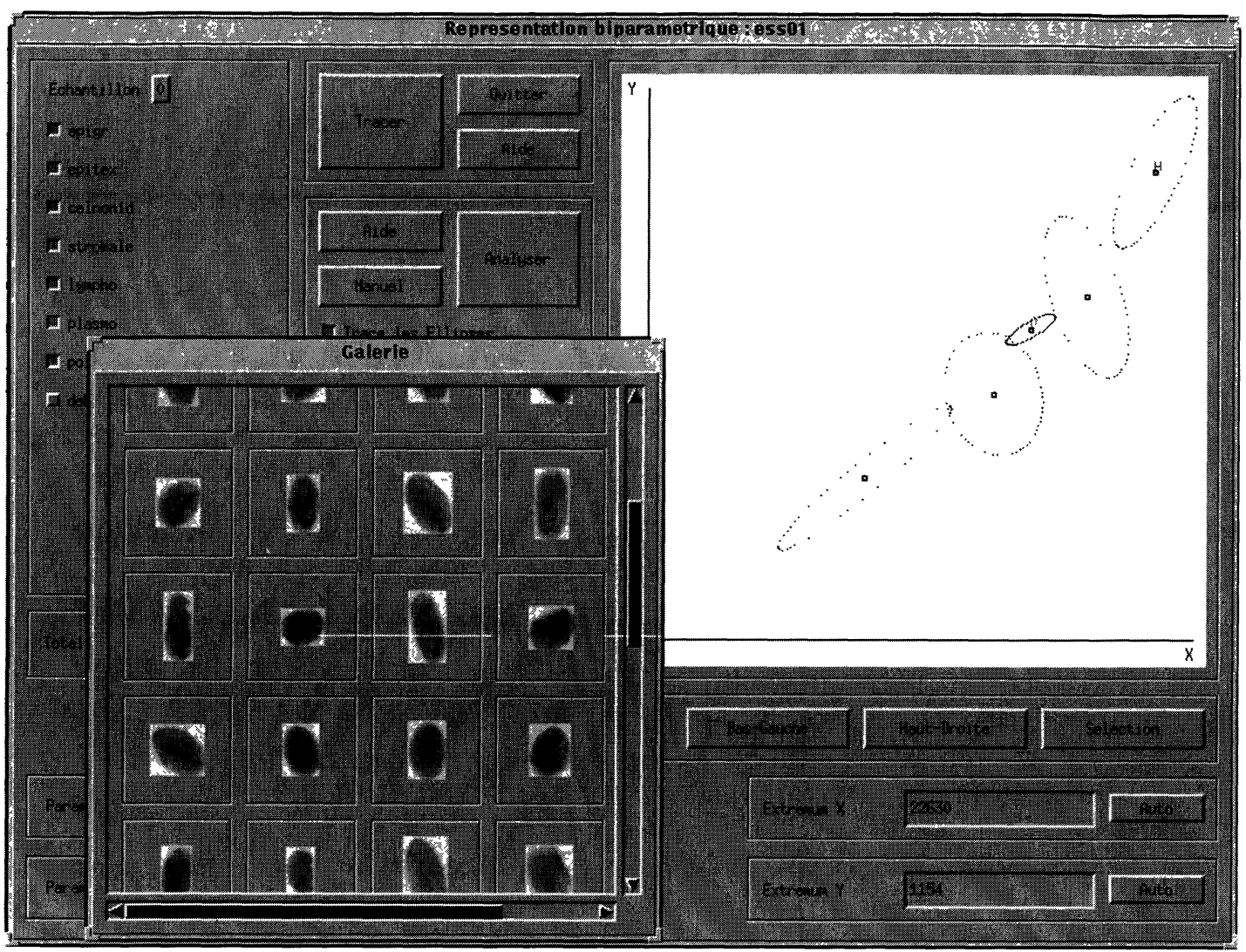

Fig. 3. - Dynamic clustering of cell populations from a 2D-map according to the choice of typical elements on an image gallery.

The visualization of a large collection of objects of interest, on a unique image, at the same resolution than the original image, greatly reduces the number of images to manipulate and treat. This can save time for the elaboration of a new image analysis strategy. Furthermore, the juxtaposition of segmented objects prior to labellisation, considerably enhances object density per image as it removes a large part of uninformative background. It is a way to save time when using mathematical morphology operators, whose time consuming is strictly dependent on the number of images to treat and independent on the number of objects per images. Two solutions are offered to treat gallery as an image: the snap shot of the gallery displayed on a screen or the successive pastings of objects in a new image. The first solution is the most quickly done and in use in the laboratory.

\section{Conclusion}

The visualization of image galleries offers to pathologists a tool for an a posteriori control of the quality of cell sorting. Apart from this already known use, pathologists can take advantages of small images by several ways: building galleries of examples, using them for rapid interactive sorting or using them to enrich their knowledge. Furthermore, image analysis experts can take 

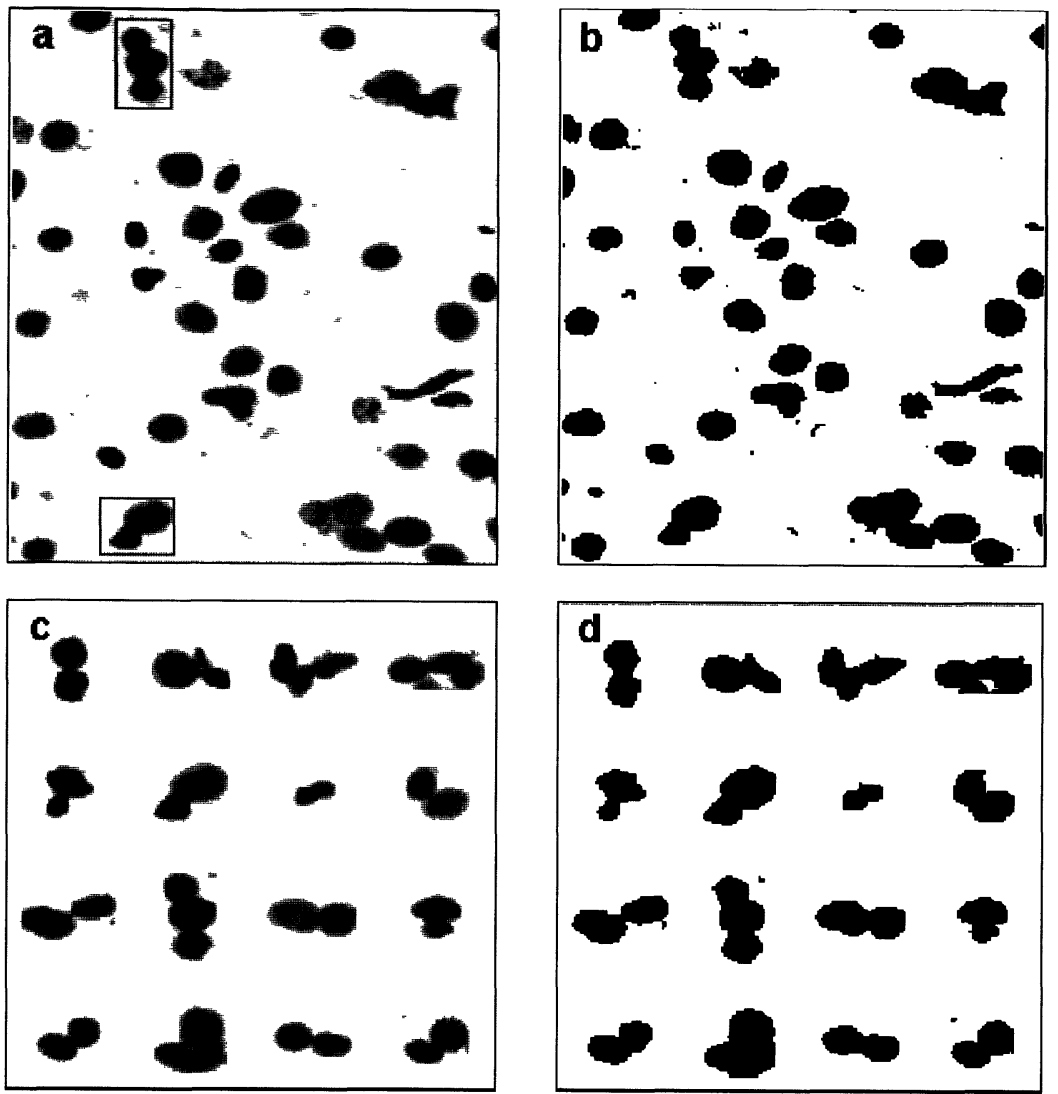

Fig. 4. - Search for aggregates of nuclei, scarce in the original images (a), in order to test image operator (b), collection of 16 aggregates in one image thanks to image galleries (c), and enriched binary image treatment facilities $(\mathrm{d})$.

advantages of this tool to build and test new strategies of analysis. Small images are an attractive tool as they are less bulky than original images and as in the future they will be easily managed in a data base together with the corresponding measurements.

\section{Acknowledgements}

This work was done under the auspices of "Pôle Traitement et Analyse d'Images (TAI) de BasseNormandie". This work was supported by grants from the "Comités Départementaux de la Ligue de Lutte contre le Cancer", from the "Association pour la Recherche sur le Cancer", from the "Fédération des Groupements des Entreprises de la Lutte Contre le Cancer", from the "Agence Nationale de Valorisation de la Recherche", from the "Centre National de la Recherche Scientifique" and from the "Conseil Régional de Basse-Normandie. Christophe Boudry and François Angot are fellows of the "Ministère de l'Enseignement Supérieur et de la Recherche". 


\section{References}

[1] Baak J.P.A., Manual of quantitative pathology in cancer diagnosis and prognosis (Springer-Verlag, Berlin, Heidelberg, 1991).

[2] Marchevsky A.M. and Bartels P.H., Image analysis. A primer for pathologists (Raven Press, New York, 1994).

[3] Duigou F., Galle I., Herlin P. and Mandard A.M., Optimization of the preparation of isolated nuclei from archival material for automatic acquisition and sorting by image analysis, Analyt. Cell. Pathol. 6 (1994) 216-217. 